\title{
Non-local conservation in the coupling field: effect on critical dynamics
}

\author{
Parongama Sen \\ Department of Physics, Surendranath College, 24/2 Mahatma Gandhi Road, Calcutta 700009, India
}

\begin{abstract}
We consider the critical dynamics of a system with an $n$-component non-conserved order parameter coupled to a conserved field with long range diffusion. An exponent $\sigma$ characterizes the long range transport, $\sigma=2$ being the known locally conserved case. With renormalisation group calculations done upto one loop order, several regions are found with different values of the dynamic exponent $z$ in the $\sigma-n$ plane. For $n<4$, there are three regimes, I: nonuniversal, $\sigma$ dependent $z$, II: universal with $z$ depending on $n$ and III': conservation law irrelevant, $z$ being equal to that in the nonconserved case. The known locally conserved case belongs to regions I and II.
\end{abstract}

Conservation laws play significant roles in critical dynamics in thermodynamic systems. The dynamics of such systems have been studied over the last several decades [1] 3]. These conservations, as constraints, generally distinguish the various dynamic universality classes for a given equilibrium class. For example, in Ising ferromagnets the order parameter may not be conserved (model A of ref. [2]) though it is so for a binary mixture (model B). In antiferromagnets or binary alloys undergoing an order-disorder transformation, a secondary parameter, the total magnetization, is conserved (model $\mathrm{C}$ ) [2, 1 . These classes are characterized by the dynamic exponent $z$ that connects the divergence of the time $(\tau)$ and length $(\xi)$ scales near the critical point, $\tau \sim \xi^{z}$. For several cases 1, 1,2,5] renormalisation group arguments give exact result for the dynamic exponent $z$. However, the numerical studies give somewhat different values which do not always agree with each other [6,7].

Most of the earlier studies assume local conservation of the conserved field, though local conservation is not a necessary, but a sufficient, condition for conservation in general. Recently the issue of non-local conservation has been addressed in the case of conserved order parameter (model B) either by relaxing local diffusion or allowing infinite range transport [8 13]. For the nonconserved case, numerical simulations have shown that the dynamic exponent can depend on the nature of the dynamics also [14. It is therefore important to determine the robustness of the wellknown dynamic universality classes.

Allowing long range diffusion in the Ising model means that the Kawasaki spin exchange now takes place with further neighbour spins also. The case where the range of the exchange becomes infinite corresponds to what we shall call "global" conservation. For the conserved order parameter case, model $\mathrm{B}$, the effect of global conservation seems to be drastic. In fact the dynamic correlation functions of the $q$ state Potts model has been shown to be

\footnotetext{
${ }^{1}$ The equilibrium exponents $\alpha, \nu$ and $\eta$ are obtained from the scaling of the specific heat $\left(\sim|r|^{-\alpha}\right)$, correlation length $\left(\sim|r|^{-\nu}\right)$, and correlation function $\left(\sim|\mathbf{x}|^{-(d-2+\eta)}\right.$ at $\left.r=0\right)$ respectively, where $r$ is the deviation from a critical point.
}

analogous to that the Ising model with fixed magnetisation and long range interactions (i.e., essentially model B with non-local conservation) [10]. Dynamics for local and global conservation are also expected to be different [13]. For $O(n)$ symmetric order parameter, the conservation law has been found to be irrelevant for global conserved case (i.e. infinite range Kawasaki dynamics) [1, 8] and the dynamic exponent $z$ is argued to be the same as that of model A. The situation is expected to be more subtle when a conserved quantity is coupled to a nonconserved order parameter as for example in model $\mathrm{C}$.

In the case of model $\mathrm{C}$, the dynamics of the nonconserved order parameter is slowed down compared to model A because of its coupling to a scalar nonordering conserved field. Even though the conserved field is not critical, its coupling to the order parameter induces long range correlations. These singular correlations in turn affect the relaxational dynamics, yielding $z_{A}<z_{C}<z_{B}$ where the subscript denotes the model name. With local conservation, model $\mathrm{C}$ has several regions in the $d-n$ plane (where $d$ is the spatial dimension and $n$ the number of components of the order parameter). There are three different regions corresponding to different values of the exponent $z$. Above $n=4$, the behaviour is model A-like with $z=2+c \eta$, i.e, there is correction to $O\left(\epsilon^{2}\right),{ }^{1}$ where $\epsilon=4-d$. For $2<n<4$, the values of $z$ are $2+2 \alpha /(n \nu)$, and $z=2+\alpha / \nu$ for $n<2$, where $\alpha$ and $\nu$ are respectively the specific heat and the correlation length exponents. (These values of $z$ are for $\alpha>0$, otherwise one takes $\alpha=0$ and $z$ once again becomes $z_{A}$.) The early time behaviour of dynamical models with short range initial correlations also gives a new exponent 15 18. Now, the emergence of the long range correlation in the conserved field, the hallmark of model $\mathrm{C}$, is an equilibrium property. Then, are the universal dynamic behaviours, as reflected in the $d-n$ plane, sensitive to the nature of the dynamics? We study this problem by considering 
a long range transport for the conserved field, a convenient way of interpolation between the local to the global conservation.

Let us consider the dynamics of an $n$ component order parameter field $\phi$ coupled to a scalar noncritical but conserved field $m$ which may be the energy density or the magnetisation in the Ising antiferromagnetic case or particle density in the binary alloy case, or annealed mobile impurities. We allow for subdiffusive or superdiffusive transport of the conserved field. This means long range transport for particles or the impurities [19] or long range exchange of spins in magnetic systems. The Hamiltonian for model $\mathrm{C}$ reads,

$$
\begin{array}{r}
H=\int d^{d} \mathbf{x}\left[\frac{1}{2} r \phi^{2}(\mathbf{x})+\frac{1}{2}(\nabla \phi(\mathbf{x}))^{2}+\tilde{u} \phi^{4}(\mathbf{x})\right. \\
\left.+\gamma \phi^{2}(\mathbf{x}) m(\mathbf{x})+\frac{1}{2} C^{-1} m^{2}(\mathbf{x})\right]
\end{array}
$$

where, $\phi^{2}(\mathbf{x})=\sum_{i=1}^{n} \phi_{i}^{2}(\mathbf{x}), \phi^{4}(\mathbf{x})=\left[\sum_{i=1}^{n} \phi_{i}^{2}(\mathbf{x})\right]^{2}, r=$ 0 is the mean field critical point, and $C>0$ ensures noncriticality of $m$.

For purely relaxational dynamics, the equation of motion obeyed by $\phi$ is

$$
\frac{1}{\Gamma} \frac{\partial \phi_{\alpha}(\mathbf{x}, t)}{\partial t}=-\frac{\partial H}{\partial \phi_{\alpha}(\mathbf{x}, t)}+\eta_{\alpha}(\mathbf{x}, t),
$$

while $m$ satisfies the following dynamical equation in Fourier space

$$
P(k) \frac{\partial m(\mathbf{k}, t)}{\partial t}=-\frac{\partial H}{\partial m(-\mathbf{k}, t)}+\zeta(\mathbf{k}, t)
$$

with the noise obeying

$$
\begin{aligned}
&\langle\zeta\rangle=0,\left\langle\eta_{\alpha}\right\rangle=0 \\
&\left\langle\eta_{\alpha}(\mathbf{x}, t) \eta_{\alpha^{\prime}}\left(\mathbf{x}^{\prime}, t^{\prime}\right)\right\rangle=2 \Gamma \delta\left(\mathbf{x}-\mathbf{x}^{\prime}\right) \delta\left(t-t^{\prime}\right) \delta_{\alpha, \alpha^{\prime}} \\
&\left\langle\zeta(\mathbf{k}, t) \zeta\left(\mathbf{k}^{\prime}, t^{\prime}\right)\right\rangle=2 P(k) \delta\left(\mathbf{k}+\mathbf{k}^{\prime}\right) \delta\left(t-t^{\prime}\right) .
\end{aligned}
$$

For model $\mathrm{C}$ with local conservation one chooses, $P(k)=$ $1 /\left(\lambda k^{2}\right)$. Here we use the general form [8]

$$
P(k)=\left[\frac{1}{\lambda_{\sigma} k^{\sigma}}+\frac{1}{\lambda_{0}}\right]
$$

For conservation to hold one strictly needs to satisfy the continuity equation only. For local conservation, the additional assumption is that the current is determined by the local change in the chemical potential (this is the case when $\sigma=2$ and Fick's law is obeyed, i.e, $j(\mathbf{x}) \propto \nabla \mu(\mathbf{x})$, where $j$ is the current density and $\mu(\mathbf{x})$ is the chemical potential in the particle picture). When the conservation is non-local, Fick's law is no longer valid. This is analogous to the invalidity of Ohm's law for diffusing electrons following Levy flight instead of nearest neighbour hopping 20]. In the long range diffusion case, instead of Fick's law, one can have a more general constitutive equation $j(\mathbf{x}) \propto \int \kappa\left(\mathbf{x}-\mathbf{x}^{\prime}\right) \nabla \mu\left(\mathbf{x}^{\prime}\right) d \mathbf{x}^{\prime}$ (which gives back Fick's law for $\left.\kappa=\delta\left(\mathbf{x}-\mathbf{x}^{\prime}\right)\right)$. This for a power law decay of $\kappa(\mathbf{x})$ on Fourier transformation gives Eq. (2b) with Eq. $(3 \mathrm{~d})$. We further include the momentum independent term, a kinetic coefficient, $\lambda_{0}$ which is generated by the RG itself. A value of $\sigma \neq 2$ simulates the nonlocality. The only constraint on $\sigma$ to ensure conservation is $\sigma>0$. The limit $\sigma \rightarrow 0^{+}$is the case corresponding to global conservation.

We use the momentum shell renormalisation group method where the Fourier components $\phi_{k}(t)$ for the hard modes $\Lambda / b<k<\Lambda$ are eliminated and the scale changes $k=k / b$ and $t=b^{z} t$ are made. We eventually set $\Lambda=1$. The long distance long time results are obtained from the flow of the renormalized parameters. The static fixed points (at one loop) of this model are easily obtained $(\epsilon=4-d)[5]$ as follows:

$$
\begin{aligned}
r^{*} & =\frac{1}{2} \frac{n+2}{n+8} \epsilon \\
u^{*} & =\frac{\epsilon}{4 K_{d}(n+8)}
\end{aligned}
$$

(here $u=\tilde{u}-\frac{1}{2} \gamma^{2} C$, and $\left.K_{d}=2^{1-d} \pi^{-d / 2} \Gamma(d / 2)\right)$.

$$
\left(\gamma^{2} C\right)^{*}=\frac{\tilde{\alpha}}{2 n \nu K_{d}}
$$

And either $\gamma^{*}=0$ or

$$
\tilde{\alpha} / \nu=(4-n) \epsilon /(n+8) ; \quad \gamma^{*} \neq 0
$$

$\tilde{\alpha}$ is the exponent of the temperature dependence of the largest term in the specific heat, i.e, $\tilde{\alpha}=\alpha$ if $\alpha>0$ and $\tilde{\alpha}=0$ otherwise ( $\alpha$ is the specific heat exponent). For $n>4, \gamma^{*}=0$ is the only fixed point. In general, $\gamma^{*}=0$ is the fixed point for $n>n_{c}$ where $n_{c}$ also has an $\epsilon$ expansion.

Considering the various vertex functions, the renormalisation equation for the transport and kinetic coefficients can be obtained [5]. The RG transformation being analytic cannot renormalise the singular terms of $P(k)$, as in the local case. However the transport coefficient generates (and renormalizes) the kinetic coefficient, $\lambda_{0}$, and also renormalizes the kinetic coefficient of the order parameter. Defining the dimensionless parameter $\mu_{\sigma}=\Gamma C / \lambda_{\sigma}$, the recursion relations at the static fixed point of Eq. (4d) can be written as (choosing thin shells $b=1+\delta l$ with $\delta l \rightarrow 0$ )

$$
\begin{aligned}
\frac{\partial \Gamma^{-1}}{\partial l} & =\Gamma^{-1}\left[-z+2+\frac{2 \tilde{\alpha} / \nu n}{\left(1+\mu^{-1}\right)}\right] \\
\frac{\partial \lambda_{0}^{-1}}{\partial l} & =\lambda_{0}^{-1}\left(-z+\tilde{\alpha} / \nu+\frac{2 n \gamma^{2} \lambda_{0} K_{d}}{\Gamma}\right) \\
\frac{\partial \mu_{\sigma}}{\partial l} & =\mu_{\sigma}\left[\sigma-2+\tilde{\alpha} / \nu-\frac{2 \tilde{\alpha} / \nu n}{1+\mu^{-1}}\right] .
\end{aligned}
$$

Here $\mu=\mu_{\sigma}+\mu_{0}$, with $\mu_{0}=\frac{\Gamma C}{\lambda_{0}}$. The above one loop contributions in the dynamic quantities come from the $\gamma$ vertex. (It maybe noted that we have excluded $\eta$ as 
$\eta=0$ to first order in $\epsilon$. This is not crucial as there is a cancellation in model $\mathrm{C}$ in higher order terms [5.24.)

The dynamic exponent $z$ is chosen to keep $\Gamma$ scale invariant. Eq. (5a) then gives

$$
z=2+\frac{2 \tilde{\alpha} / \nu n}{\left(1+\mu^{-1}\right)} .
$$

The fixed point of $\mu_{0}$ is obtained from eqs. (5b) and (4a)

$$
\mu_{0}^{*}=\frac{\tilde{\alpha} / \nu}{2(z-\tilde{\alpha} / \nu)}
$$

The fixed points of $\mu_{\sigma}$ are therefore $0, \infty$ or a non-zero finite value given by

$$
\mu_{0}^{*}+\mu_{\sigma}^{*}=\frac{\sigma-2+\tilde{\alpha} / \nu}{2-\sigma+\frac{\tilde{\alpha}}{\nu} \frac{2-n}{n}}
$$

Stability of fixed points and exponents:

Let us analyse the stability of the fixed points for the different values of $\sigma$.

Above $n=4, \gamma^{*}=0$ is the only fixed point and therefore the conserved field gets decoupled in long lengthscale limit. This is nothing but the model A fixed point where the ordering and non-ordering fields are no longer coupled and therefore $z=z_{A}$. To recover $z_{A}=2+c \eta$ one needs to go to higher loops, where the $u$ vertex starts contributing.

$\mu_{\sigma}=0$ is the fixed point valid for $\sigma<2-\alpha / \nu$ and the exponent $z=2+O\left(\epsilon^{2}\right)$ for all $n$. At this fixed point the conservation of the coupling field simply becomes irrelevant. One therefore again recovers model A exponent $z=z_{A}$, although $\gamma^{*} \neq 0$.

We note that as $\mu_{\sigma}^{*}$ cannot be negative and $\tilde{\alpha} \sim O(\epsilon)$, (7) will give a physical fixed point only if $n<2 /(1+p)$ where $p=(\sigma-2) /(\tilde{\alpha} / \nu)$ and $p>-1$. For $4>n>$ $2 /(1+p), p>-1, \mu_{\sigma}=\infty$ will be the stable fixed point. The latter gives $n=4$ at $p=-0.5$, so for $-1<p<-0.5$, we find that a non-zero finite fixed point for $\mu_{\sigma}^{*}$ is always stable. In particular, for $\sigma=2$, we get back the results of [2]: $z=2+\tilde{\alpha} / \nu$ for $n<2, z=2+2 \tilde{\alpha} / n \nu$ for $2<n<4$ and $z=2+O\left(\epsilon^{2}\right)$ for $n>4$.

Thus we have the following regions in the $\sigma-n$ plane:

I. $z=\sigma+\tilde{\alpha} / \nu$.

For

$\sigma=2+p \tilde{\alpha} / \nu, n<2 /(1+p) ; p>-1$

II. $z=2+2 \tilde{\alpha} / n \nu$.

For

$\sigma=2+p \tilde{\alpha} / \nu, 4>n>2 /(1+p) ; p>-1 / 2$

III. $z=2+O\left(\epsilon^{2}\right), \gamma^{*}=0$.

For all $\sigma, n>4$.

III' $z=2+O\left(\epsilon^{2}\right), \gamma^{*} \neq 0$.
For $\sigma<2-\alpha / \nu \quad$ (i.e., $p=-1$ ), $n<4$.

These are shown schematically in Fig. 1. We may add that there are discontinuities of $z$ only at the boundaries $\sigma=2-\tilde{\alpha} / \nu$ and $n=4$. The model $\mathrm{C}$ like regions for $\sigma=2$ are in regions I and II with nothing special for the $\sigma=2$ line. The value of $z$ is also continuous across the boundary of regions I and II. Of the two regions, I is nonuniversal with a $\sigma$ dependent dynamic exponent $z=\sigma+\tilde{\alpha} / \nu$ while II is robust and independent of $\sigma$. In region II, $z$ is dependent on $n$ explicitly while in region I, $n$ dependence is through the equilibrium exponents only. As one crosses the $\sigma=2$ line, region II extends towards smaller values of $n$ for $\sigma>2$ and the opposite happens for $\sigma<2$ where it vanishes at $n=4, \sigma=2-\tilde{\alpha} / 2 \nu$ and region I survives as long as $\sigma>2-\tilde{\alpha} / \nu$.

The general $\sigma$ case, in addition, allows for a new regime where the conservation law becomes irrelevant even though the coupling survives $(\gamma \neq 0)$ in the long length scale limit and induces long range correlations in equilibrium. The global conservation case belongs to this class.

Since the nonrenormalizability of $\lambda_{\sigma}$ is valid to all orders, we expect, as in the local model $\mathrm{C}$, the dynamic exponent $z$, as obtained here, for regions I and II to be exact. The above analysis is best for $d$ close to 4 as we have done calculations upto $O(\epsilon)$ only. For $d$ close to 4 , in fact, region I exists for values of $\sigma \geq 2$ and $\sigma<2$ and very close to 2 only as $\alpha \sim O(\epsilon)$. Continuing with this result in lower dimensions, one expects region I to exist over a larger range of $\sigma(>2)$ so that the line separating regions I and II in the positive half of the $\sigma-2$ axis in Fig. 1 goes higher up for lower dimensions. However, the line separating regions II and III (or III' and III) in Fig 1 is shifted towards lower values of $n$ for lower dimensions as $\alpha$ becomes negative at a critical value of $n=n_{c}$ where $n_{c}$ also has an $\epsilon$ expansion. (Fig. 1 has been drawn for $d$ close to 4 ). In the $d-n$ plane therefore, region I is in general depleted for a value of $\sigma$ higher than 2. This depletion is maximum for $d=4$. For $\sigma<2-\tilde{\alpha} / \nu$, only region III or III' survive with $z=z_{A}$ for all $d$.

Some of our results are in contrast with the long range diffusion in model $\mathrm{B}$, where $z$ is explicitly dependent on $\sigma$ as long as the conservation is relevant. The dynamical exponent is in general expected to increase for a system with constraint, and therefore $z \geq z_{A}$. However, global conservation (i.e., $\sigma \rightarrow 0^{+}$) becomes irrelevant and one recovers $z=z_{A}$ in this limit [8, 12, 11]. This would the case for $\sigma<\sigma_{c}=z_{A}-2+\eta=O\left(\epsilon^{2}\right)$ for model B. In model $\mathrm{C}$, we find region III', where conservation is irrelevant, to be significantly larger with $\sigma_{c}=2-\tilde{\alpha} / \nu$. Even when the conservation is relevant, we find a strongly universal regime, region II, where the conservation slows down the dynamics but the nature of the dynamics is irrelevant ( $\sigma$ independence).

It is possible to implement this long range transport in a simulation. The infinite range would correspond 
to $\sigma \rightarrow 0^{+}$, while any other $\sigma$ can be implemented by choosing the two spins to be flipped, in the Kawasaki dynamics, at a distance $l$ with probability $P(l) \sim l^{-(r+\sigma)}$ for large $l$. However, it is fairly easy to identify the irrelevance of the conservation in model $\mathrm{B}$ numerically when the model $\mathrm{A}$ exponent is recovered, as $z_{A}$ and $z_{B}$ are easily distinguishable even with moderate level of precision in calculations. For model C, very high precision simulations are required to verify our RG based scenario as the model $\mathrm{C}$ exponents in region I and II are different from that of model A by a much lesser margin.

To summarise our results, we find the effect of long range diffusion in model $\mathrm{C}$ with an $n$-component order parameter to be highly significant. Characterising the long range diffusion by an exponent $\sigma>0$, several regimes with different dynamical exponents $z$ are obtained in the $\sigma-n$ plane. For $n<4$, a region with a $\sigma$ dependent $z$ is found to exist as well as a region with strong universality where $z$ is independent of $\sigma$. Most remarkable is the existence of a region for $\sigma$ greater than a critical value where the coupling with the field survives in the large length scale limit but the conservation becomes irrelevant and $z=z_{A}$. Such a region is also present in model B with non-locally conserved order parameter, although for a much smaller range of $\sigma$. For $n>4$, the coupling with the field is irrelevant and $z=z_{A}$ as expected. The known results for the locally conserved model $\mathrm{C}$ are recovered with $\sigma=2$.

[1] A. J. Bray, Adv. Phys. 43, 357 (1994).

[2] P. C. Hohenberg, B. I Halperin, Rev. Mod. Phys. 49435 (1977).

[3] J. D. Gunton, M. San Miguel ans P. S. Sahni in Phase Transitions and Critical Phenomena, Vol 7, ed. C. Domb and J. L. Lebowitz (Academic Press, London 1983).

[4] E. Eisenriegler and B. Schaub, Z. Phys. B 3965 (1980).

[5] P. C. Hohenberg, B. I. Halperin and S. K. Ma, Phys. Rev. B 10139 (1974), ibid 134119 (1976), E. Brezin and C. De Domonicis Phys. Rev. B 124954 (1975).

[6] P. Sen, S. Dasgupta and D. Stauffer, Eur. Phys. J. B 1 107 (1998).
[7] D. Stauffer, Int. J. Mod. Phys C 81263 (998).

[8] A. J. Bray, Phys. Rev. B 416724 (1990); Phys. Rev. Lett. 62, 2841 (1989).

[9] P. Tamayo and W. Klein, Phys. Rev. Lett. 63. 2757 (1989).

[10] C. Sire and S. N. Majumdar, Phys. Rev. E 52244 (1995).

[11] L. L. Moseley, P. W. Gibbs and N. Jan, J. Stat. Phys. 67, 813 (1992)

[12] J. F. Annett and J. R. Banavar, Phys. Rev. Lett. 68, 2941 (1992).

[13] A. D. Rutenberg, Phys. Rev. E 54972 (1996).

[14] J. Salas and A. D. Sokal, J. Stat. Phys. 85297 (1996).

[15] B. Zheng, Int. J. Mod. Phys. B 121419 (1988).

[16] K. Oerding and H. K. Janssen, J. Phys. A 263369 (1993)

[17] J. Cardy, J. Phys. A 252765 (1992)

[18] S. Mukherji, cond-mat/9809354, to appear in Eur. Phys. J. B.

[19] J. P. Bouchaud and A. Georges, Phys. Rep. 195127 (1990).

[20] V. E. Arkhincheev and A. V. Nomeev, preprint (1998).

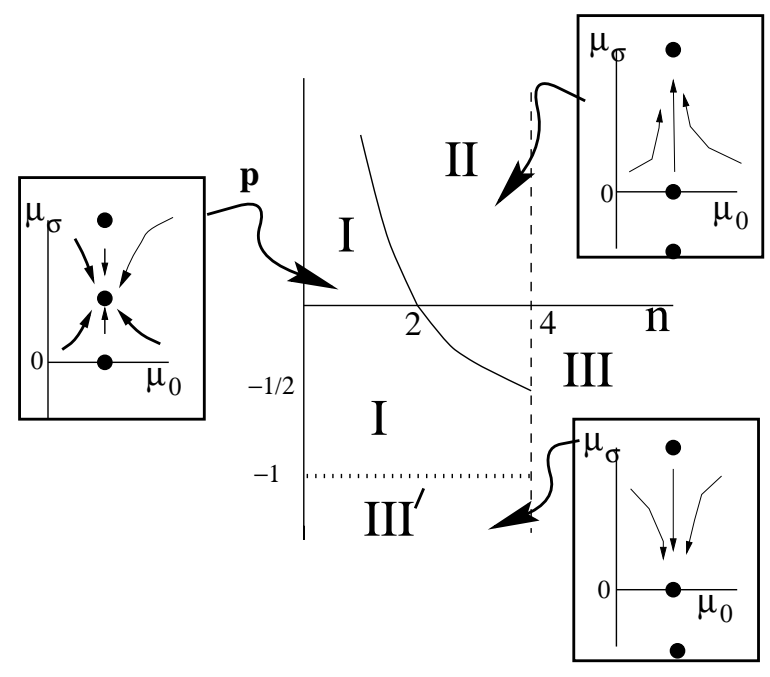

FIG. 1. Regions corresponding to different values of $z$ are shown for $p=\frac{\sigma-2}{\tilde{\alpha} / \nu}$ and $n$. The flow diagrams in $\mu_{\sigma}-\mu_{0}$ plane are also shown. The flow is towards a finite non-zero value of $\mu_{\sigma}$ in region I, towards $\mu_{\sigma} \rightarrow \infty$ in region II and $\mu_{\sigma}=0$ in region III'. 